\title{
Task-shifting to optimize outpatient neurological care in Zambia
}

\author{
Ana C. Villegas' ${ }^{1}$ Deanna Saylor ${ }^{2,3}$, Michelle Kvalsund ${ }^{2,4}$, Masharip Atadzhanov², Clarence Chiluba ${ }^{2}$, \\ Lorraine Chishimba ${ }^{2}$, Stanley Zimba ${ }^{2}$, Mashina Chomba ${ }^{2}$ and Omar K. Siddiqi ${ }^{2,5,6^{*}}$ (D)
}

\begin{abstract}
Objective: To investigate opportunities for task shifting to decongest an outpatient neurology clinic in Zambia by describing current patient flow through the clinic and potential nodes for intervention using process mapping.

Background: Zambia has a population of approximately 18 million people with 4 full-time adult neurologists, as of 2018, who all practice at the University Teaching Hospital (UTH), the main tertiary care center in the country. As a result of this provider-to-patient ratio, the outpatient neurology clinic is overcrowded and overbooked. Task-shifting programs have shown to improve efficiency, access and quality of care through the use of less specialized healthcare workers in low- and middle-income countries (LMIC).

Methods: We evaluated patient flow in the UTH neurology outpatient clinic through the development and analysis of a process map. The characteristics of the clinic population between 2014 and 2018 were retrospectively reviewed from the clinic register. Between July and August 2018, we prospectively collected appointment lag times and time each patient spent waiting at various points in the clinic process. We conducted interviews with clinic staff and neurologists to generate a detailed process map of current pathways to care within the clinic. We then devised taskshifting strategies to help reduce patient wait times based on the overview of clinic process mapping and patient demographics.
\end{abstract}

Results: From 2014 to 2018, there were 4701 outpatients seen in the neurology clinic. The most common neurological diagnoses were epilepsy (39.2\%), headache (21.5\%) and cerebrovascular disease (16.7\%). During prospective data collection, patients waited an average of 57.8 (SD 73.4) days to be seen by a neurologist. The average wait time from arrival in the clinic to departure was 4.0 (SD 2.5) h. The process map and interviews with clinic staff revealed long waiting times due to a paucity of providers. Nurses and clerks represent an influential stakeholder group, but are not actively involved in any activity to reduce wait times. A large proportion of follow-up patients were stable and seen solely to obtain medication refills.

Conclusions: Epilepsy, headache, and stroke make up the largest percentage of outpatient neurological illness in Zambia. Targeting stable patients in these diagnostic categories for a task-shifting intervention may lead to substantially decreased patient wait times. Potential interventions include shifting clinical follow-ups and medication refills to less specialized healthcare workers.

Keywords: Task-shifting, Nurses, Neurology, Sub-Saharan Africa, Zambia

*Correspondence: osiddiqi@bidmc.harvard.edu

${ }^{2}$ Department of Internal Medicine, University of Zambia School of Medicine, Lusaka, Zambia

Full list of author information is available at the end of the article

\section{Background}

Neurological disorders represent a costly global public health problem and account for $10.2 \%$ of the global disability-adjusted life years (DALYs) and $16.8 \%$ of global original author(s) and the source, provide a link to the Creative Commons licence, and indicate if changes were made. The images or other third party material in this article are included in the article's Creative Commons licence, unless indicated otherwise in a credit line to the material. If material is not included in the article's Creative Commons licence and your intended use is not permitted by statutory regulation or exceeds the permitted use, you will need to obtain permission directly from the copyright holder. To view a copy of this licence, visit http://creativecommons.org/licenses/by/4.0/. The Creative Commons Public Domain Dedication waiver (http://creativeco mmons.org/publicdomain/zero/1.0/) applies to the data made available in this article, unless otherwise stated in a credit line to the data. 
deaths [1]. With an increasingly older population coupled with limited access to neurological care, the burden of neurological disease in low- and middle-income countries (LMIC) is predicted to continue to increase with a projected 103 million DALYs in 2030 [1].

Previous research has estimated that the average ratio of neurologists to the general population in Africa is on the order of 1:3.4 million, with 11 nations without any neurologists [2]. In comparison, there are between 4 and 13 neurologists per 100,000 people in Europe and the US [3, 4]. Zambia is located in southern Africa and has a high burden of neurological disease with marked delays in accessing neurological care. Its estimated population in 2016 was $\sim 18$ million [5] with 4 adult neurologists all of whom practice at University Teaching Hospital (UTH), the national referral hospital, in the capital city of Lusaka. As a result of this providerto-patient ratio, the outpatients:neurologist ratio is extremely high (Table 1 ).

The World Health Organization (WHO) has expressed a need to address under-diagnosis and prompt treatment of non-communicable chronic conditions, recognizing that the model of specialist physician management is not feasible in resource-poor settings [6]. Task-shifting programs have been shown to improve efficiency, access and quality of care through the use of less specialized healthcare workers in specific settings in Africa. This approach is best characterized in HIV/AIDS care but has never been applied to neurological care in LMIC. There needs to be a better understanding of appropriate delegation of tasks by non-specialists in specific populations to successfully implement task-shifting in neurological care. Evidence can help inform strategies to in order to promote better access to neurological care in Africa.

To address this clinical and research gap, we collected essential information on the outpatient neurology clinic at UTH. This study attempted to identify areas to improve efficiency within the UTH outpatient neurology clinic. Based on these findings, we propose an initial frame work for task shifting to aid implementation of neurological care in resource-limited settings.

\section{Methods}

UTH is Zambia's national referral hospital and is located in the capital city of Lusaka. It is the main teaching hospital for the University of Zambia School of Medicine. The UTH outpatient neurology clinic is staffed by four neurologists who, during the study period, were the only practicing neurologists in the country. This clinic has a neurophysiology laboratory that offers electroencephalograms (EEG) and electromyography/nerve conduction (EMG/NCS) studies. Providers refer patients to the UTH outpatient neurology clinic for specialty evaluation, after hospitalization for a neurological disorder, or for neurophysiology testing.

We employed a mixed quantitative and qualitative approach as there were no national pathways or processes for task-shifting initiatives to guide professionals in neurological care at the time the study was conducted. We elected to use process mapping which incorporates the patient experience to improve the quality or efficiency of clinical management [7]. It is a tool that has shown clinical benefit when applied to healthcare systems $[7,8]$.

The procedure has been used as a knowledge translation strategy for health systems research in Africa [9]. In Ghana and in South Africa, process mapping has been used as a strategy to combat lung cancer and hypertension $[10,11]$. Our approach was novel because process mapping has been used to shape policy change in population health, but has never been used to analyze neurological care. We sought to use process mapping as a quality improvement (QI) tool in order to decrease hurdles associated with implementation of neurological care in resource-limited settings and facilitate further quality improvement efforts.

The team consisted of a Master of Public Health graduate student and two neurologists providing outpatient care in Zambia to review care-delivery processes. We conducted standardized in-person field interviews with clinic staff regarding workflow using a standardized interview guide with question prompts. Data gained from these interviewers were then used to develop process maps. Eligible staff who agreed to participate in the quality improvement research project were English-speaking

Table 1 Provider-to-patient ratio at the outpatient neurology clinic, 2014-2018

\begin{tabular}{lllllll}
\hline Year & $\mathbf{2 0 1 4}$ & $\mathbf{2 0 1 5}$ & $\mathbf{2 0 1 6}$ & $\mathbf{2 0 1 7}$ & $\mathbf{2 0 1 8}$ & Total \\
\hline Patients & 86 & 737 & 1022 & 1103 & 1753 & 4701 \\
Neurologists & 1 & 2 & 2 & 2 & 0.002 & 0.002 \\
Neurologist-to-patient ratio & 0.011 & 0.002 & 0.002 & $16,853,599$ & $17,943,895$ \\
Zambian population & $15,399,788$ & $15,879,361$ & $16,363,458$ & & 3 \\
\hline
\end{tabular}

This study only collected data from 3 out of the 4 neurologists currently working at UTH 
as is standard in Zambia. We conducted interviews during periods chosen by staff so as not to interfere with clinical duties. During the staff interviews, staff members provided input to process maps to ensure accounting for all tasks involved in clinical care. We then developed a point-by-point checklist of the process from start to finish (Table 2). Based on the information from the process maps, we prospectively collected appointment lag time periods (e.g., time between initial referral to neurology clinic and first consultation in the clinic) and the total time patients spent waiting in the clinic (e.g., time from patient arrival at clinic to being called into the examination room to see a neurologist) between July and August 2018. Neurological complications of HIV infections are common, even in patients on effective antiretroviral therapy. Since task-shifting interventions in Africa have been primarily studied in the context of HIV care provision

Table 2 A point-by-point checklist of the patient process from start to finish at the neurology outpatient clinic

\begin{tabular}{lll}
\hline Tasks & Completed $(\mathrm{Y} / \mathrm{N})$ & Time \\
\hline Checklist & & \\
Scheduling & & \\
Arrival & & \\
Front desk & \\
Vital signs & \\
Examination room & \\
\hline
\end{tabular}

[12-15], we analyzed these data separately based on patient's HIV status.

Patients were not directly interviewed. Instead, they were given a written survey by clinic staff to carry during the visit upon arrival to the registration desk. As patients moved through the clinic, the study team recorded the time at which each stage of the outpatient process (e.g., registration, vitals, visit with the doctor, etc.) began. Once the visit was complete, patients returned the questionnaire to the registration desk. Quantifying these times on the process map, allowed us to quantitatively analyze the time spent on each step of the procedure and identify inefficiencies for potential improvement. We held online team debriefings to discuss the process map results. This allowed all the team members to identify any further problems and provide suggestions.

In addition to the process map and wait times, we reviewed the outpatient log of all patients seen by the three neurologists in the clinic from 2014 to 2018. We placed all patients into categories based on their diagnosis at the time of their visit (Table 3). A number of cases did not have a final diagnosis due to resource limitations. Based on the number of patients in each diagnostic category, we were able to identify high yield neurological conditions for efficiency initiatives.

\section{Results}

The annual outpatient visit frequency and prevalence of HIV infection seen at the UTH outpatient neurology clinic over the last 4 years are presented in Fig. 1.

Table 3 Detailed description of the most common disease categories seen at the neurology outpatient clinic

\begin{tabular}{|c|c|}
\hline Disease category & Definition \\
\hline Seizures/epilepsy & Patients who have suffered a seizure or carry the diagnosis of epilepsy \\
\hline Infectious & $\begin{array}{l}\text { Patients whose primary presentation was related to an underlying infection of the nervous system such as cryptococ- } \\
\text { cal meningitis, toxoplasmic encephalitis, or cerebral malaria }\end{array}$ \\
\hline Headache/cephalgias & Primary headache disorders such as tension, migraine, and cluster headaches or some related disorder \\
\hline Neuromuscular & Disorders of the neuromuscular junction, muscle, or motor neuron disease \\
\hline Movement disorders & Patients with tremor, Parkinson's disease, ataxia or other related disorders \\
\hline Neuropathy/radiculopathy & $\begin{array}{l}\text { A heterogenous group of peripheral nervous system disorders including diabetic neuropathy, AIDP/CIDP, plexopathies, } \\
\text { and radiculopathies }\end{array}$ \\
\hline Cerebrovascular disease & Patients with ischemic stroke, hemorrhagic stroke, and/or transient ischemic attacks \\
\hline Myelopathy & $\begin{array}{l}\text { A broad category that included patients with obvious signs of spinal cord dysfunction such as leg weakness, bowel/ } \\
\text { bladder symptoms, or sensory level without any further details to clarify the diagnosis }\end{array}$ \\
\hline Dementia/neurodegenerative & Patients with AIDS-Dementia Complex, Alzheimer's disease, Huntington's disease, and/or cerebellar degeneration \\
\hline Demyelinating & $\begin{array}{l}\text { It was restricted to the central nervous system and included cerebral demyelination, acute disseminated encephalomy- } \\
\text { elitis, and transverse myelitis }\end{array}$ \\
\hline Encephalopathy & A cognitive dysfunction of unknown origin whose time course fit an acute/subacute confusional state \\
\hline Diagnosis unclear & When it was unclear what a patient had, or if they could not be placed into a diagnostic category with great certainty \\
\hline Other conditions & If patients had a condition that represented $<1 \%$ of the study population \\
\hline
\end{tabular}

This diagnostic category is based on a previous study at UTH [22] 

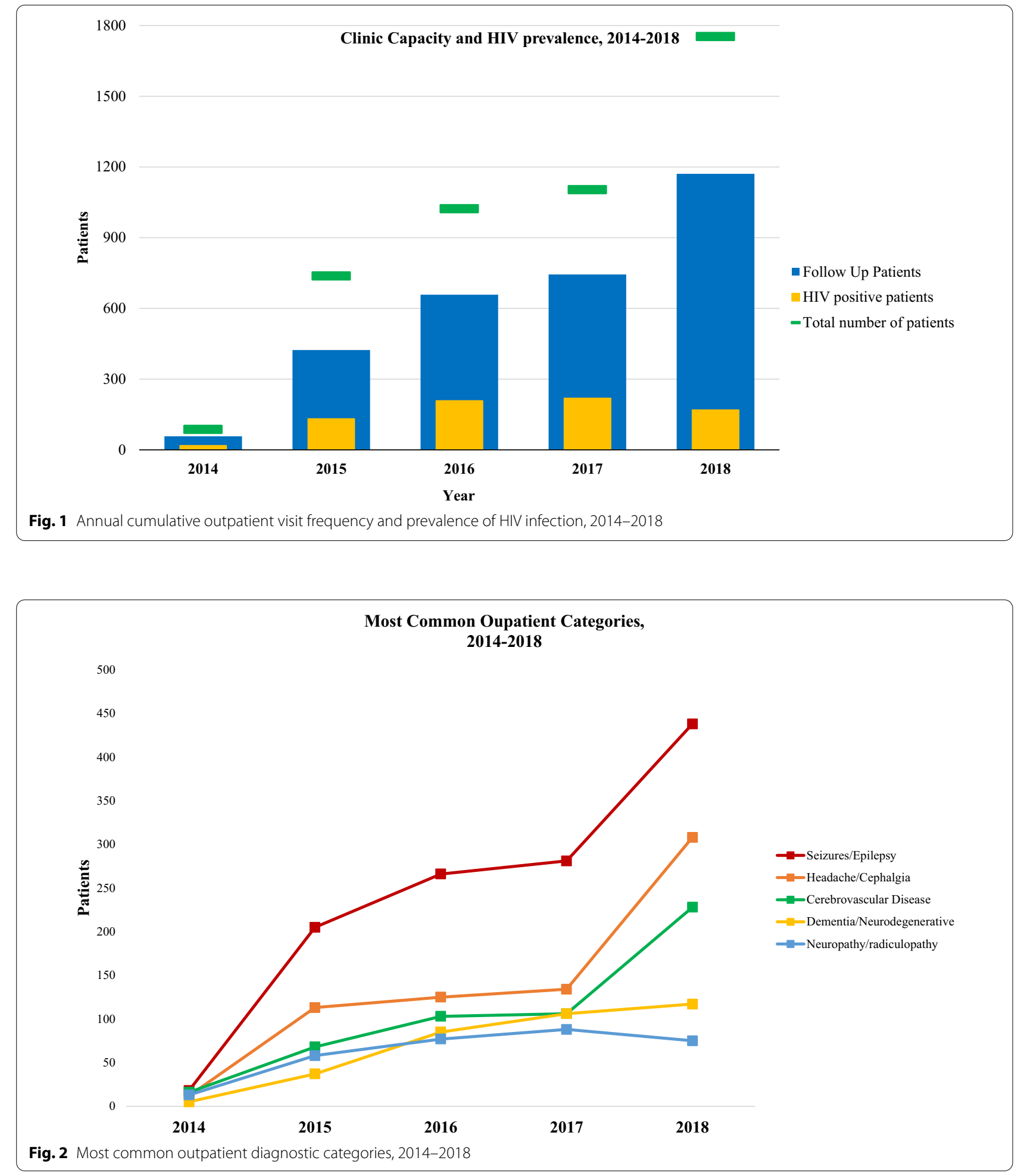

From January 2014 to December 2018, the UTH outpatient neurology clinic had 4701 visits, of those 1648 (35.0\%) were new patients and 3053 (65.0\%) were followup patients. The highest volume year was 2018 which included 1753 visits. The median age of patients seen at the outpatient neurology clinic was 42.9 years. A total of $883(19.0 \%)$ were people living with HIV, accounting for $396(27.5 \%)$ of all male patients and 487 (20.5\%) of all 


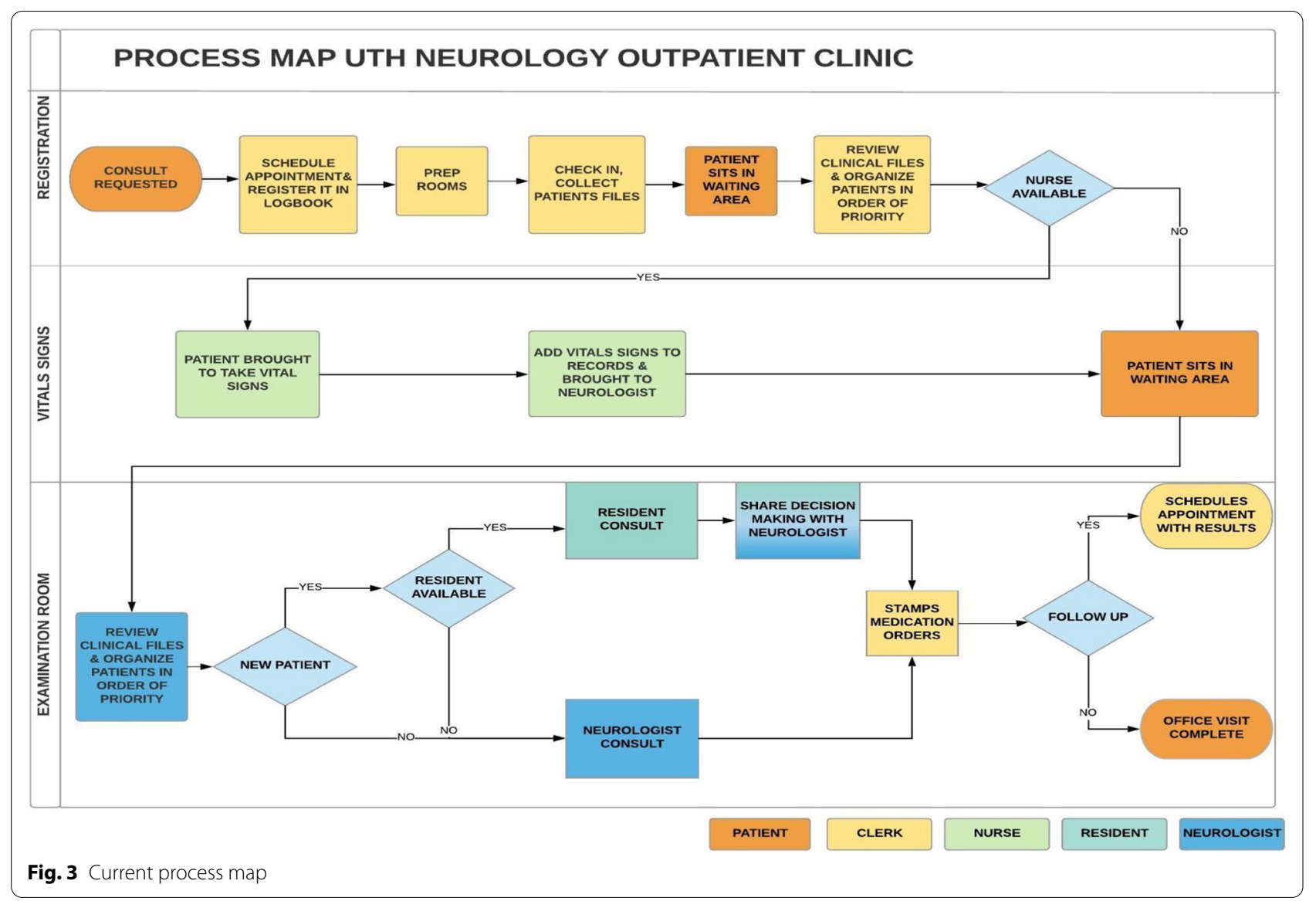

female patients cared for at the clinic. Figure 2 shows the outpatient neurologic diagnostic categories seen from 2014 to 2018 with epilepsy (39.2\%), headache $(21.5 \%)$ and stroke $(16.7 \%)$ as the most common.

Figure 3 provides the finalized process map based on interviews with staff from the UTH outpatient neurology clinic. The process map detailed a challenge for each of the three main steps of the patient flow through the UTH outpatient neurology clinic: registration, recording vital signs, and examination.

The registration desk is the first point of contact for the patients at the UTH outpatient neurology clinic, which runs on a first come, first served basis. Patients start to arrive before its opening to be seen by the team of neurologists as early as possible. Over the course of the study, each physician sees 24.5 patients per week. Of those, only $30 \%$ of the patients scheduled an appointment. Once a patient is registered, files are transitioned in the same room to the nurses' station.

At the nursing station, the nurse takes the patient's vital signs in the order of arrival. The files are then transferred to the examination rooms. Based on the debriefing sessions with clinic staff and the neurology team, nurses and clerks represent an influential stakeholder group from the registration area. Because of their constant role at the frontline, their practices have a decisive impact. However, often times they were not being effectively utilized. According to the field data, they are not actively involved in any activity to reduce waiting times.

Debriefing with clinic staff and neurologists also revealed consensus that long wait times tend to aggravate patients and their families. Examples that were cited include that often stable epilepsy, headache and stroke patients have to wait between 3 and $4 \mathrm{~h}$ to obtain a medication refill. They further reported that a large proportion of follow-up patients in these diagnostic categories were stable and largely seen in clinic solely to obtain medication refills. Neurologists also reported that they assume full responsibility for these delays, which leaves them feeling overextended in attempts to see and treat every single patient that presents to clinic that day. As a result, they reported that they commonly see patients on non-clinic days as a strategy to decongest and reduce the workload on clinic days.

Patient-reported appointment lag times and clinic wait times are reported in Table 4. On average, patients waited 
Table 4 Patient-reported times waiting and getting into the clinic

\begin{tabular}{|c|c|c|c|c|}
\hline \multirow[t]{2}{*}{ Steps } & \multicolumn{2}{|l|}{ New patient } & \multicolumn{2}{|l|}{ Follow-up patient } \\
\hline & Minutes median & IQR & Minutes median & IQR \\
\hline Appointment lag time (days) & 30 & 39 & 120 & 129 \\
\hline Getting to the clinic & 40 & 40 & 40 & 37.5 \\
\hline 1) Arrival to the clinic (h) & 7:15 AM & $0: 56$ & 7:00 AM & $1: 16$ \\
\hline 2) Front desk to vital signs & 74 & 88 & 67 & 41.5 \\
\hline $\begin{array}{l}\text { 3) Hand-off clinical files until in examination room } \\
\text { with neurologists }\end{array}$ & 96 & 198 & 147 & 125 \\
\hline
\end{tabular}

IQR Interquartile range. 1) Statistics are based on 41 patients over 3 Clinic sessions. 2) All figures are median (IQRS) unless otherwise specified. 3) Not all patients had their vital signs taken $(N=9)$

57.8 (SD 73.4) days to be seen by a neurologist. The average wait time in clinic was 4.0 (SD 2.5) h.

\section{Discussion}

This study provides valuable information about neurological services at the national referral hospital in Zambia. Consistent with prior studies from the region, seizure, headache and stroke are the most common diagnoses seen at the UTH outpatient neurology clinic $[16,17]$. Stable patients in the top three diagnostic categories, requiring only medication refills, were determined to be the optimal target for a task-shifting intervention to reduce patient wait times. Programs targeting medication refills have been shown to improve adherence and decrease physician workload [18-20]. Figure 4 illustrates the recommended approach to the

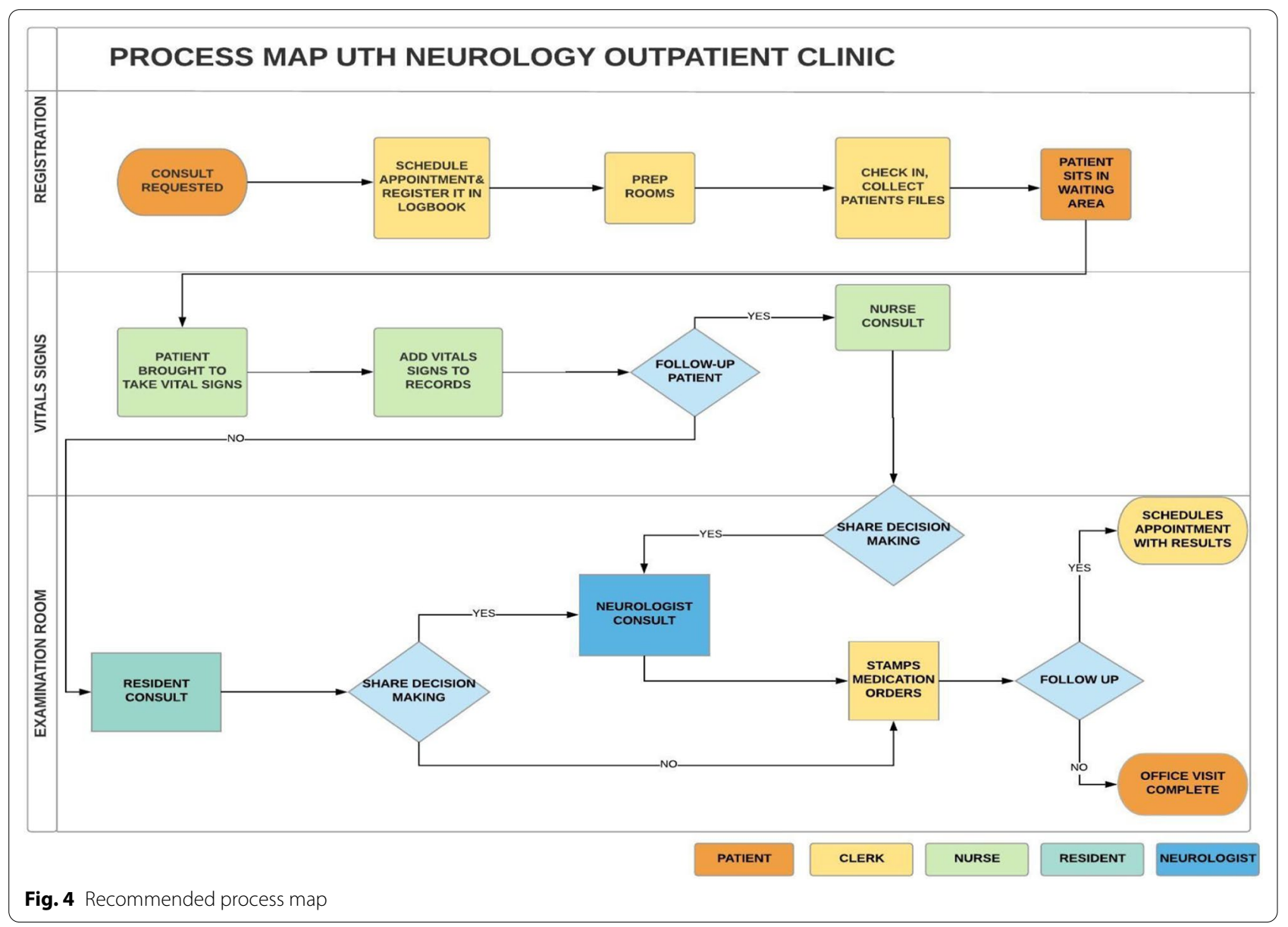


processes around the UTH outpatient neurology clinic, outlining a starting point for effective execution of the task-shifting program in neurology.

Neurology guidelines for upper and middle-income economies recommend individual patient management delivered by neurologists prescribing treatment, supported by imaging and high-technology laboratory work monitoring [21]. Unfortunately, this approach in Zambia and in other LMIC is often not feasible due to lack of neurologists, diagnostic investigations and effective treatments. In fact, the neurological exam remains the most useful diagnostic tool [22]. Our study found that long waiting times for patients to see a neurologist and scarce neurology workforce are adding to the current shortfall. In fact, the UTH outpatient neurology clinic has increased from 1 to 4 neurologists during the study period. In 2018, they experienced the highest patient volume, which likely resulted from the addition of the fourth neurologist. The demand for neurologists, however, continues to exceed supply. Unfortunately, these shortages are likely to be sustained. Thus, guidelines are not translating into clinical practice in these settings because it is not realistic [23]. Alternative strategies to improve access to care for patients with neurological care are urgently needed in LMIC.

Task-shifting to nurses and community health workers may benefit numerous components of the neurology care delivery in LMIC. In Mozambique, training nonphysician clinicians in a task-shifted model of antiretroviral therapy rollout for persons living with HIV, compared to physician care, was able to improve health outcomes including viral suppression, toxicity and mortality [24, 25]. Building upon previous findings, we believe common outpatient conditions such as epilepsy, headache and stroke, may benefit from protocols that make it easier for less specialized healthcare workers to manage these conditions.

In this specific context, we recommend shifting clinical follow-ups and refills of chronic medications to nurses, pharmacists and EEG/EMG technicians. These less specialized healthcare workers, however, have had limited training in managing these disorders, so implementation would require an adequate planning and training. A task-sharing model could be adopted first followed by task-shifting as non-physician healthcare workers gained more experience and confidence with their new responsibilities. Ideally, community healthcare workers will be able to determine whether these patients are truly stable and then take accurate treatment decisions. To do so, it is necessary to create a clinical environment that is sufficiently regular and predictable (i.e., only performing clinical evaluations of the same conditions and patient population) because it gives nurses and community health workers the opportunity to learn these conditions through prolonged practice [26]. As the healthcare workers gain more confidence in deciding whether someone is stable or not, the task could be completely shifted to them to identify stable patients without routine discussion with the neurologist.

It is important to note that strengthening training without changes in systems such as clinical operations and procedures, may lead to missing unstable patients, and thus may prove insufficient to improve patient care and/or outcomes [27]. Conversely, we believe that to maximize the accuracy of clinical evaluations made by community healthcare workers, final neurological assessments and decisions should be supported by a checklist of the most common conditions seen at the UTH outpatient neurology clinic. Research has shown that the use of checklists improves clinical outcomes and involves both changes in systems and in the individual and clinical teams' behavior [28]. The virtues of checklists are well documented in LMIC, where community healthcare workers were able to substantially improve their performances of measure practices with the use of a checklist, as well as improving patient morbidity rates [29].

Based on the Consolidated Framework for Implementation Research (CFIR) [30] creating tension for change is necessary and feasible through better data collection. We encouraged the UTH outpatient neurology clinic to include key performance indicators (kpi): new visit rate, new appointment lag time and no-show rate. These kpis are measurable values that demonstrate how effectively a clinic is achieving the neurology task-shifting goals and overall healthcare objectives. These measures will allow evaluation of this approach, not only for baseline but for ongoing monitoring of the task-shifting program, as well as garner support from leadership. With the creation of urgency, elevating the matter to an organizational aim, there will be an increased awareness among providers, and therefore a stronger culture to improve neurological care using differentiated models of care. Task-shifting can have a relatively quick effect on utilization and on new appointment lag time by increasing the capacity of the services. Hence, there will be a need for shorter evaluation cycles of this neurology task-shifting model, that focuses on process optimization, avoiding radical workflow and operational changes. Task-shifting interventions in HIV populations in Africa may be used as a benchmark for comparing efficiency in a potential task-shifting intervention in neurology in similar countries.

This study has several limitations. The current study only considered the view of patients and providers in a national referral hospital and not populations in more rural settings in Zambia. Since our study is limited in its geographic reach, the findings may therefore be 
biased towards this population's experiences and preferences. However, process mapping is a method that can be adapted and used to identify gaps in other outpatient neurology clinics in LMICs, which in turn can help those clinics to tailor the use of differentiated models of care to meet their identified needs.

In addition, our data brought to light other issues in the clinic such as the low appointment bookings rate, which raises the question of what communication barriers patients are experiencing when trying to book appointments in advance. Unfortunately this information was not collected as it was out of the scope of the project. These issues warrant further consideration and may pose opportunities to better communicate with patients by reinforcing the need to schedule appointments in advance or allowing patients to schedule appointments with the use of mobile apps and other technologies. Finally, this study focuses on a clinic in which fully trained neurologists are available to evaluate new patients and support community healthcare workers participating in the task-shifting models. Our suggestions would likely not apply to less-resourced settings in which no neurologists were present.

In Zambia, as happens in other LMICs, the treatment gap of neurological disorders is due in part to a small or absent neurology workforce leading to long waiting times to meet the growing demand for their services. Prospective solutions to reduce lag times may include task-shifting neurological care to nurses and community health workers. This approach will require training, so less specialized healthcare workers can develop the skills needed to identify and make the most appropriate referrals to neurologists and to resume management of stable neurology patients.

\section{Abbreviations \\ UTH:The University Teaching Hospital; LMIC: Low- and middle-income coun- tries; DALYs: The global disability-adjusted life years; NCS: Nerve conduction;} EEG: Electroencephalograms; EMG: Electromyography studies.

\section{Acknowledgements}

The authors would like to thank the staff in the neurology clinic at the University Teaching Hospital as well as the patients and their families.

\section{Authors' contributions}

OKS and ACV were key conceivers and designers of the study methodology. ACV performed, signed and conducted the interviews, led the study point prevalence analysis and the process map-based analysis of interview data. She also led the writing of the manuscript. OKS, DS and MK participated in the process mapping, consensus reaching and provided critical revisions to the manuscript intellectual content. All authors read and approved the final manuscript.

\section{Funding}

The content is solely the responsibility of authors and does not represent the official views of any funding source. Sponsors had no role in the design, analysis, interpretation, or publication of the study.

\section{Availability of data and materials}

Raw interview data are not publicly available due to them containing information that could compromise participants privacy. Derived datasets used and/ or analyzed during the current study are available from the corresponding author on reasonable request.

\section{Declarations}

Ethics approval and consent to participate

The study was designed as a quality improvement at the University Teaching Hospital outpatient neurology clinic. The project was approved by the Managing Director of the University Teaching Hospital and the Department of Internal Medicine, University of Zambia School of Medicine, Lusaka, Zambia. The ethical approval of the project and the manuscript submission was provided by the Zambia National Health Research Association through an approval letter that can be provided upon request.

\section{Consent for Publication}

Not applicable.

\section{Competing interests}

The authors declare that they have no competing interest.

\section{Author details}

${ }^{1}$ School of Public Health, Boston University, Boston, MA, USA. ${ }^{2}$ Department of Internal Medicine, University of Zambia School of Medicine, Lusaka, Zambia. ${ }^{3}$ Department of Neurology, Johns Hopkins University School of Medicine, Baltimore, MD, USA. ${ }^{4}$ International Neurologic \& Psychiatric Epidemiology Program, Department of Neurology \& Ophthalmology, Michigan State University, East Lansing, MI, USA. ${ }^{5} \mathrm{Global}$ Neurology Program, Department of Neurology, Beth Israel Deaconess Medical Center, Harvard Medical School, 330 Brookline Avenue, Boston, MA 02215, USA. ${ }^{6}$ Center for Virology and Vaccine Research, Department of Internal Medicine, Beth Israel Deaconess Medical Center, Harvard Medical School, Boston, MA, USA.

Received: 30 June 2020 Accepted: 10 June 2021

Published online: 16 July 2021

\section{References}

1. Group GBDNDC. Global, regional, and national burden of neurological disorders during 1990-2015: a systematic analysis for the Global Burden of Disease Study 2015. Lancet Neurol. 2017;16(11):877-97.

2. Chomba EN, Haworth A, Mbewe E, Atadzhanov M, Ndubani P, Kansembe $\mathrm{H}$, et al. The current availability of antiepileptic drugs in Zambia: implications for the ILAE/WHO "out of the shadows" campaign. Am J Trop Med Hyg. 2010;83(3):571-4.

3. Dall TM, Storm MV, Chakrabarti R, Drogan O, Keran CM, Donofrio PD, et al. Supply and demand analysis of the current and future US neurology workforce. Neurology. 2013;81(5):470-8.

4. Freeman WD, Vatz KA, Griggs RC, Pedley T. The Workforce Task Force report: clinical implications for neurology. Neurology. 2013;81(5):479-86.

5. United Nations Population Division. World Population Prospects: The 2017 Revision. 2016 July 13, 2018.

6. World Health Organization. Task shifting to tackle health worker shortages. 2007.

7. Trebble TM, Hansi N, Hydes T, Smith MA, Baker M. Process mapping the patient journey: an introduction. BMJ. 2010;341:C4078.

8. King DL, Ben-Tovim DI, Bassham J. Redesigning emergency department patient flows: application of Lean Thinking to health care. Emerg Med Australas. 2006;18(4):391-7.

9. Edwards A, Zweigenthal V, Olivier J. Evidence map of knowledge translation strategies, outcomes, facilitators and barriers in African health systems. Health Res Policy Syst. 2019;17(1):16.

10. Menyanu E, Baatiema L, Charlton K, Wilson M, Aikins AD, Russell J, et al. Towards population salt reduction to control high blood pressure in Ghana: a policy direction. Curr Dev Nutr. 2020;4(Suppl 3):nzaa084. 
11. Dlamini SB, Sartorius B, Ginindza T. Mapping the evidence on interventions to raise awareness on lung cancer in resource poor settings: a scoping review protocol. Syst Rev. 2019;8(1):217.

12. Babigumira JB, Castelnuovo B, Stergachis A, Kiragga A, Shaefer $P$, Lamorde $M$, et al. Cost effectiveness of a pharmacy-only refill program in a large urban HIV/AIDS clinic in Uganda. PLoS ONE. 2011;6(3):e18193.

13. Tran A, Thakur KT, Nakasujja N, Nakigozi G, Kisakye A, Batte J, et al. Evaluation of a screening tool for the identification of neurological disorders in rural Uganda. J Neurol Sci. 2021;421:117273.

14. Saylor D. Neurologic complications of human immunodeficiency virus infection. Continuum (Minneap Minn). 2018;24(5):1397-421.

15. Holroyd KB, Vishnevetsky A, Srinivasan M, Saylor D. Neurologic complications of acute HIV infection. Curr Treat Options Infect Dis. 2020;12(3):227-42.

16. Kengne AP, Anderson CS. The neglected burden of stroke in Sub-Saharan Africa. Int J Stroke. 2006;1(4):180-90.

17. Akinyemi RO, Owolabi MO, Ihara M, Damasceno A, Ogunniyi A, Dotchin $C$, et al. Stroke, cerebrovascular diseases and vascular cognitive impairment in Africa. Brain Res Bull. 2019;145:97-108.

18. Gross R, Zhang Y, Grossberg R. Medication refill logistics and refill adherence in HIV. Pharmacoepidemiol Drug Saf. 2005;14(11):789-93.

19. Nguyen M, Zare M. Impact of a Clinical Pharmacist-Managed Medication Refill Clinic. J Prim Care Community Health. 2015;6(3):187-92.

20. Matlin OS, Kymes SM, Averbukh A, Choudhry NK, Brennan TA, Bunton A, et al. Community pharmacy automatic refill program improves adherence to maintenance therapy and reduces wasted medication. Am J Manag Care. 2015;21(11):785-91.

21. Berkowitz AL, Westover MB, Bianchi MT, Chou SH. Aspirin for acute stroke of unknown etiology in resource-limited settings: a decision analysis. Neurology. 2014;83(9):787-93.
22. Siddiqi OK, Atadzhanov M, Birbeck GL, Koralnik IJ. The spectrum of neurological disorders in a Zambian tertiary care hospital. J Neurol Sci. 2010;290(1-2):1-5

23. Berkowitz AL. Managing acute stroke in low-resource settings.; 2016.

24. Callaghan M, Ford N, Schneider H. A systematic review of task-shifting for HIV treatment and care in Africa. Hum Resour Health. 2010;8:8.

25. Nelissen C, Sherriff J, Jones T, Guest P, Colley S, Sanghera P, et al. The Role of Positron Emission Tomography/Computed Tomography Imaging in Head and Neck Cancer after Radical Chemoradiotherapy: a Single Institution Experience. Clin Oncol (R Coll Radiol). 2017;29(11):753-9.

26. Kahneman D, Egan P. Thinking, fast and slow. Farrar SaG, editor. New York 2011

27. Dettrick Z, Firth S, Jimenez SE. Do strategies to improve quality of maternal and child health care in lower and middle income countries lead to improved outcomes? A review of the evidence. PLOS ONE. 2013:8(12):e83070.

28. Haynes AB, Weiser TG, Berry WR, Lipsitz SR, Breizat AH, Dellinger EP, et al. A surgical safety checklist to reduce morbidity and mortality in a global population. N Engl J Med. 2009;360(5):491-9.

29. Semrau KEA, Hirschhorn LR, Marx Delaney M, Singh VP, Saurastri R, Sharma N, et al. Outcomes of a Coaching-Based WHO Safe Childbirth Checklist Program in India. N Engl J Med. 2017;377(24):2313-24.

30. Keith RE, Crosson JC, O'Malley AS, Cromp D, Taylor EF. Using the Consolidated Framework for Implementation Research (CFIR) to produce actionable findings: a rapid-cycle evaluation approach to improving implementation. Implement Sci. 2017;12(1):15

\section{Publisher's Note}

Springer Nature remains neutral with regard to jurisdictional claims in published maps and institutional affiliations.
Ready to submit your research? Choose BMC and benefit from:

- fast, convenient online submission

- thorough peer review by experienced researchers in your field

- rapid publication on acceptance

- support for research data, including large and complex data types

- gold Open Access which fosters wider collaboration and increased citations

- maximum visibility for your research: over $100 \mathrm{M}$ website views per year

At BMC, research is always in progress.

Learn more biomedcentral.com/submissions 